\title{
The probability-outcome correspondence principle: A dispositional view of the interpretation of probability statements
}

\author{
GIDEON KEREN \\ Eindhoven University of Technology, Eindhoven, The Netherlands \\ and \\ KARL HALVOR TEIGEN \\ University of Tromsø, Tromsø, Norway
}

\begin{abstract}
This article presents a framework for lay people's internal representations of probabilities, which supposedly reflect the strength of underlying dispositions, or propensities, associated with the predicted event. From this framework, we derive the probability-outcome correspondenceprinciple, which asserts that strong dispositions should lead to (1) strong (forceful) and (2) immediate outcomes and, hence, be characterized by high probabilities. In contrast, weak dispositions lead to (1) weak (fragile) and (2) delayed outcomes and are thus associated with low probabilities. We describe six experiments designed to test the correspondence principle. In the final discussion, we examine the implications of the proposed framework, from both a normative and a descriptive viewpoint.
\end{abstract}

The concept of probability is the most common device by which uncertainty can be analyzed, expressed, and communicated. The concept is embedded in a formal theory, in which probability is the only undefined primitive (Salmon, 1967). As a branch of mathematics, the formal calculus of probability is undisputed (Shafer, 1993), yet the meaning and possible interpretations of the probability concept remain subject to an ongoing controversy from both normative (Salmon, 1967) and descriptive (Wright \& Ayton, 1994) viewpoints.

Among the main rival normative explications of the concept of probability are the classical (based on the notion of equally likely events), the frequentistic (which defines probability in terms of the relative frequency of the occurrence of an attribute or an event approaching an infinite number of observations), the logical (where probability is regarded as a logical relation between propositions that formulate evidence and hypotheses whose truth is not fully determined by the evidence), and the subjective, in which probability is interpreted as a measure of degree of belief (Salmon, 1967, is recommended for an illuminating discussion). A fifth interpretation of probabilities as representing propensities has been proposed by Popper $(1959,1990)$ and is briefly discussed below.

With the exception of the subjective view, all the other interpretations are detached from direct psychological and behavioral considerations. During the past 3 decades, there

This research was partly supported by a grant from the Research Council of Norway to K.H.T. Correspondence concerning this article should be addressed to G. Keren, Faculty of Technology Management, Eindhoven University of Technology, TEMA Building 0.08, P.O.B. 513, 5600 MB Eindhoven, The Netherlands (e-mail: g.b.keren@tm.tue.nl). have been several attempts to further elaborate on the psychological aspects associated with probabilistic statements. For example, Howell and Burnett (1978) proposed a distinction between internal and external uncertainties, depending on whether the outcomes are controllable or not. Kahneman and Tversky (1982) applied the same terms, respectively, to distinguish between states of uncertainty that are solely determined by internal reflections of the mind (and are thus by definition subjective), and states of uncertainty that are attributed to the external world and can supposedly be assessed in more objective ways (e.g., by using frequency distributions). These authors also offered subdivisions of the internal-external distinction, which primarily refer to the type of evidence employed for advocating a particular probability statement. Teigen (1994) adopted a similar approach but proposed a different subdivision of the external-internal distinction.

The intricate issues associated with the meanings of probability have both normative and descriptive facets. ${ }^{1}$ In the present article, we focus mainly on descriptive aspects related to the question of how people interpret probability statements. We believe that people's assessments of probabilities are not just derived from specific computational rules or from particular heuristics. To understand how people interpret probability statements, it may be useful to examine how they conceive probabilities in the broadest sense. To that end, we are searching for a lay theory of probability that will be similar to lay theories of physics (e.g., McCloskey, 1983) or to implicit personality theories (e.g., Chiu, Hong, \& Dweck, 1997). The implicit probability theory we envisage cannot be elicited directly, because people would rarely be able to formulate it explicitly. Notwithstanding, we can confirm the existence of such a 
lay theory since, as we show empirically, it manifests itself in different ways in people's judgments.

The theoretical framework that we adopt assumes that people tend to think of probabilities in causal terms, as tendencies or predispositions favoring specific outcomes. This notion bears some similarity to Popper's $(1959,1960)$ propensity view of probabilities. ${ }^{2}$ Specifically, Popper $(1959,1990)$ advanced the idea that probabilities are physical propensities comparable to Newtonian forces. Similar to a force that entails a dispositional physical entity, a propensity introduces a dispositional property of physical events, and it is this propensity that underlies a probability statement. Popper claimed that "probabilities must be 'physically real' - that they must be physical propensities, abstract relational properties of the physical situation, like Newtonian forces" (Popper, 1959, p. 28). ${ }^{3}$ Popper rejected the subjective interpretation of probabilities and insisted that uncertainty can be attributed only to the external world. Hence, he claimed that propensities are solely characteristic of the physical world. In contrast, we adopt the view that probabilities are represented mentally and, accordingly, assume the existence of counterpart propensities in lay people's conceptualization of probabilities. Moreover, in our framework, propensities encapsulated in probability statements do not necessarily have to be associated with an external measurable physical entity. We thus propose that people may think about the propensity of a student to pass an exam, the propensity of a soccer team to win a game, or the propensity encapsulated in a job candidate that, in turn, will determine the probability that she will be offered the job. ${ }^{4}$

The framework we propose entails that probabilities reflect underlying dispositions. Specifically, strong dispositions are believed to lead to strong and immediate outcomes and should be characterized by high probabilities. In contrast, weaker dispositions may lead to less conspicuous and more delayed outcomes and should be characterized by lower probabilities. We label this phenomenon the principle of probability-outcome correspondence.

Underlying the correspondence principle is the observation, originally developed by John Stuart Mill, that people tend to assume that causes and effects should be of similar magnitude (Mill, 1856; see also Medin, 1989). The principle can manifest itself in a number of ways. For single outcomes, high-probability values may be seen as corresponding to high-amplitude outcomes (e.g., a high probability of an earthquake may be interpreted not only as predicting an earthquake for sure, but as predicting a particularly strong earthquake). The probability magnitude can also be reflected in temporal characteristics of the predicted event, so that the higher the probability, the sooner the event should occur. It may also be reflected in the frequency of the event: The higher the probability, the more often it should occur. This is, of course, identical to the basic tenet of the frequentistic view on probabilities. The difference between the frequency interpretation and the principle of probability-outcome correspondence is that we regard the latter as a more general principle that can be manifested not only in frequencies, but also in other quantifiable outcome characteristics.

The inferential reasoning underlying the correspondence principle is, in many cases, warranted. Indeed, strong causes often lead to strong effects, which may also be highly predictable. For instance, heavy rainfalls may be easier to predict (and hence be associated with higher probabilities) than traces of rain. But there may also be cases in which such a correlation is harder to justify. To take one example, a medicine with a high probability of side effects will not necessarily have more severe side effects than another drug that is claimed to have a lower probability of side effects. In short, the correspondence principle can be normatively defended under some circumstances, but not under others. However, following the dispositional lay theory, people generalize the correspondence principle also under circumstances in which it cannot be logically justified.

In a separate series of experiments (Keren \& Teigen, 2001), it was shown that people generally tend to prefer higher probabilities to lower or intermediate probabilities, presumably because higher probabilities are felt to contain more definite and unambiguous information and are thus more valuable for predictive purposes. Higher probabilities were also considered to be more "correct" than lower probabilities when the predicted outcome actually occurred. This preference for high probabilities, which we termed the principle of search for definitivepredictions, outweighed, in some cases, considerations for proper calibration (i.e., participants preferred an overconfident forecaster to a well-calibrated one).

In these previous studies, however, all the predictions concerned categorical outcomes-for example, whether it would rain or not, or whether a medical treatment would be successful or not. Under such circumstances, high probabilities can be taken as evidence of a strong tendency for the occurrence of the outcome in question and, thus, be in better correspondence with the actual outcome than are lower probabilities. In contrast, the present set of studies focuses on quantitative outcomes - that is, outcomes that are described in terms of degrees (e.g., strong vs. weak earthquakes, winning with a wide vs. a narrow margin). In this case, we can expect low probabilities to be descriptive of the occurrence of low-amplitude events. In other words, there may be occasions on which low $p$ predictions are regarded as "better" or more "correct" than high $p$ predictions, even when the predicted event actually occurs.

The following experiments were designed to test the correspondence principle in relation to quantitative outcomes under various circumstances. The methodology employed in these experiments was to provide participants with outcomes of a weak or a strong magnitude and to probe the size of the probability judgment they believed would justify each case. In accordance with the correspondence principle, we predicted that weak outcomes would be associated with low probabilities and 
strong outcomes with high probabilities. Our empirical approach differed from most previous research in that, rather than asking people to generate probability judgments, we asked them to evaluate probability judgments made by others and tested the types of information they unveiled from these judgments. Our participants were thus placed in the role of the "consumer" of probability judgments (Yates, Price, Lee, \& Ramirez, 1996), and probabilities were used as the independent (rather than the dependent) variable.

\section{EXPERIMENT 1}

The participants in Experiment 1 were asked to compare two experts who offered different probability estimates of the outcomes of four basketball games. The participants were given quantitative outcome information in terms of the exact final score of each game. The participants in one condition had to choose between high and low probabilities for games that were won with a large margin, whereas in the other condition, the games were won with a small margin. On the basis of the probability-outcome correspondence hypothesis, it was predicted that a larger number of participants would prefer low probabilities when win margins were small. Similarly, a larger number of participants would prefer high probabilities when win margins were large.

\section{Method}

Participants. The participants were 119 students from the University of Utrecht, who volunteered to participate in this and other decision-making tasks, for which they were paid 11 Dutch guilders (approximately \$6).

Design and Procedure. All the participants were presented with the same scenario, in which two basketball experts made probabilistic predictions regarding the outcomes of basketball games (the exact instructions and the different scenarios are presented in the Appendix). Both experts, named John and Peter, made the same forecasts regarding the winning team but differed in their probability estimates: For all the four games, John estimated the probability of a win for the home team to be $90 \%$, whereas Peter's probability judgments of the same outcomes were $70 \%$. The participants were also informed that the home team actually had won three out of the four games. The participants' task was to judge which of the two experts, Peter or John, made (overall) better probability forecasts.

In Condition 1, the participants were informed only about the winning team, whereas in Conditions 2 and 3, they were told the exact final scores. The difference between Conditions 2 and 3 was that, in the former, the home team won with large margins (and the single loss was based on a narrow margin), whereas in the latter, the home team won with narrow margins (and the single loss was based on a large margin). The participants were asked to judge who was the better forecaster and to briefly justify their choice.

\section{Results and Discussion}

The results are shown in Table 1 . Across the three conditions, approximately $32 \%$ of the respondents considered the two forecasters to be equally good probability forecasters. Excluding those participants, the main interest is the comparison between Conditions 2 and 3. Evidently,
John's higher probability estimates (.90) were judged to better reflect decisive wins (exhibited by large margins), as in Condition 2. In contrast, Peter's lower probability estimates (.70) were judged to better reflect minimal wins (exhibited by small margins), as in Condition 3. The difference between the number of participants preferring John over Peter in Conditions 2 and 3 is highly significant $(p<.001)$. Condition 1 , in which the exact final scores were not given, falls in this respect between Conditions 2 and 3.

An analysis of the participants' justifications for their choices was also instructive. In Condition 3 (win with small margins), 11 out of the 22 participants who preferred the lower forecast explicitly mentioned the small margin of the win. Similarly, 14 out of the 22 participants who preferred the higher forecast in Condition 2 (win with large margins) justified it by referring to the fact that only one game (out of four) was lost, and even this loss was just on the edge (i.e., with a small margin of loss). Evidently, as is predicted by the correspondence hypothesis, high probabilities were associated with decisive wins, and low probabilities were associated with marginal wins.

The above interpretation is not necessarily incompatible with formal probability theory and can, under some rigid assumptions, be explicated in formal terms. For instance, consider the basketball scenario used in Experiment 1 . There are obviously a large number of possible outcomes for each game (in terms of end results). To simplify matters, we focus on the difference in points scored by each team (e.g., a final score of 84-64 entails a difference score of 20). Consider a game between two teams, A and B. Figure 1 shows two hypothetical probability distributions of the difference score made by two forecasters, Peter and John. The 0 point on the $x$ abscissa implies a draw (no difference). The values to the right of this point indicate the difference in advantage for Team A, and those to the left for Team B. Peter predicts that Team A will win with a probability of .60 (hence, $60 \%$ of the area under his probability distribution is to the right of the 0 point). John predicts that Team A will win with a probability of 80 (hence, $80 \%$ of the area under his probability distribution is to the right of the 0 point). As can be seen from Figure 1, the probability that Team A will win with a large margin inferred from John's probability distribution is always higher than the same probability inferred from Peter's distribution. Conversely, for low difference scores, the probability

Table 1

Number of Participants (With Percentages) Preferring the Higher or the Lower Probability Judgments in the Three Conditions of Experiment 1

\begin{tabular}{|c|c|c|c|c|c|c|}
\hline \multirow[b]{2}{*}{ Condition } & \multicolumn{2}{|c|}{$\begin{array}{c}\text { Peter } \\
(p=.70) \\
\end{array}$} & \multicolumn{2}{|c|}{$\begin{array}{c}\text { John } \\
(p=.90)\end{array}$} & \multicolumn{2}{|c|}{ Same } \\
\hline & $n$ & $\%$ & $n$ & $\%$ & $n$ & $\%$ \\
\hline 1 (no end scores) & 15 & 38 & 9 & 23 & 15 & 38 \\
\hline 2 (win-large margins) & 5 & 12 & 22 & 55 & 13 & 32 \\
\hline 3 (win-small margins) & 22 & 55 & 8 & 20 & 10 & 25 \\
\hline
\end{tabular}




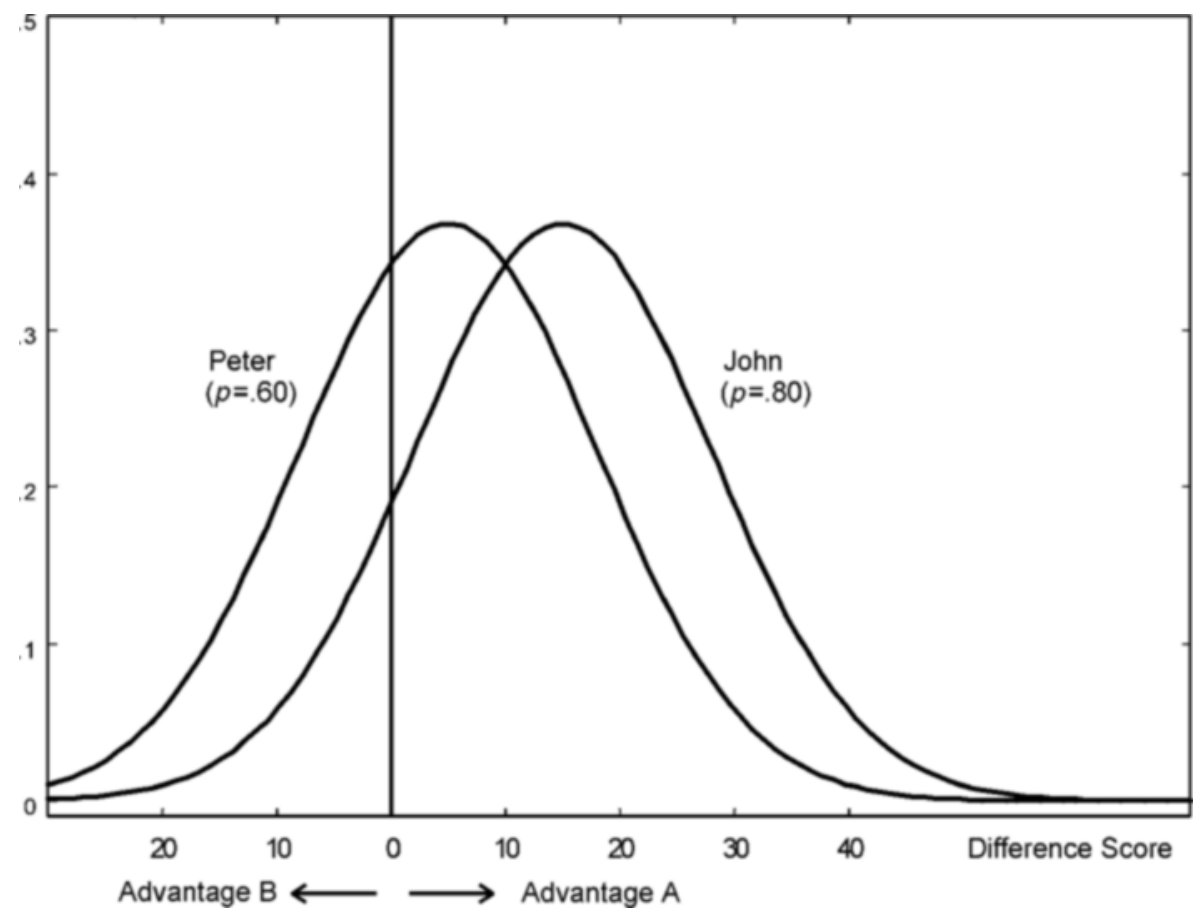

Figure 1. Hypothetical example of two probability distributions illustrating the relation between winning probability and the size of the difference score.

inferred from Peter's distribution is higher than that of John. In that respect, higher probabilities are indeed associated with larger difference scores.

\section{EXPERIMENT 2}

The results of Experiment 1 show that high probabilities correspond (in the participants' view) not only with high likelihoods, but also with the strength or impact of the outcome, as expressed in score differences. The present experiment was designed to study another measure of impact-namely, how soon the result was attained. Following the correspondence hypothesis, a team that scores early in the game should be credited with a stronger winning potential and thus obtain a higher probability of winning than would a team that scores its goals later in the game, even if the eventual scores for both teams are the same and there are no differences in the winning margin.

\section{Method}

Participants. The participants were 137 Dutch students from the University of Nijmegen, who took part in a decision-making experiment that consisted of several tasks, including the one described below. The participants were paid approximately $\$ 6$ for participating in the experiment, which lasted about $45 \mathrm{~min}$.

Materials. Three pairs of scenarios were constructed describing a forecasting competition between two soccer experts, named Mr. Peters and Mr. Landman, who made probabilistic judgments on forthcoming soccer games. According to the scenarios, both experts correctly predicted the winning team, but their probability judgments (both of which were larger than .5) differed: Mr. Peters' predictions were always associated with higher probabilities than were those of Mr. Landman. Next, there was a brief description of the game-namely, the exact times at which goals were scored. For each scenario, there were two versions: one in which the winning team scored early (i.e., the outcome was determined early in the game) and one in which the winning team scored late (just before the game was over). The participants were asked to judge which of the two forecasters made a better probability judgment (half of the participants in each scenario were asked to make a strict choice, whereas the other half had a third option of saying that both made equally good probability judgments).

In Scenarios A1 and A2, Mr. Landman predicted that Team A would win with probability of $60 \%$, and Mr. Peters estimated the probability to be $75 \%$. In both scenarios, the end result was $1-0$ for Team A except that, in the early scenario (A1), the only goal was scored in the 8th minute, whereas in the late scenario (A2), the only goal was scored in the 89th minute, just before the end of the game.

In Scenarios B1 and B2, Mr. Landman and Mr. Peters predicted that Team D would win with probabilities of $70 \%$ and $80 \%$, respectively. Team D indeed won, with a score of 3-2, but scored its goals either early or late in the game. According to the early scenario (B1), Team D was leading at halftime by $3-0$ (as a consequence of goals scored at the 3rd, 12th, and 39th minutes), an advantage that was reduced during the second half by two goals scored of the 86th and 90th minute. According to the late scenario (B2), Team D was lagging at halftime by $0-2$ but ended by winning $3-2$, owing to three goals scored at the 79th, 86th, and 90th minutes.

In the last pair of scenarios, both Mr. Landman and Mr. Peters predicted the win of Team $\mathrm{E}$ with probabilities of $75 \%$ and $90 \%$, respectively. Team E won with a score of 2-0. According to the early scenario $(\mathrm{C} 1)$, the two goals were scored at the 25 th and the 60th minutes; according to the late scenario $(\mathrm{C} 2)$, they were scored at the $81 \mathrm{st}$ and 85 th minutes. All three scenarios are presented in the Appendix. 
Design and Procedure. All the participants received exactly the same instructions (see the Appendix), followed by two scenarios. All possible combinations of two scenarios were used, with the restriction that they were never from the same pair.

\section{Results and Discussion}

The results are shown in Table 2. For the first pair of scenarios in which the game ended with a score of 1-0, the majority of the participants preferred the higher forecast (Mr. Peters, $p=.75$ ) when the winning goal was obtained early (at the 8th minute) and preferred the lower forecast (Mr. Landman, $p=.60$ ) when the winning goal was obtained late (at the 89th minute). Excluding the few participants who had no preference, the difference between the two scenarios was highly significant (using Fisher's exact test, $p<.001$ ).

This pattern of results cannot be inferred on normative grounds-there is no justification for assigning a higher probability to the earlier score. We analyzed a sample of 270 games played in the Dutch soccer leagues during 1993-1996, all of which ended with a 1-0 score. The minute at which the single goal was scored followed a uniform distribution, as was verified by a KolmogorovSmirnov test. ${ }^{5}$

A similar pattern can be observed with the second pair of scenarios. The majority of participants judged the higher probability (Mr. Peters, $p=.80$ ) to be more appropriate when the winning team led early in the game and kept the lead despite two goals scored close to the end of the game. In contrast, when the other team led $0-2$ at half-time and the win was realized only toward the end of the game, the majority opted for the lower probability judgment (Mr. Landman, $p=.70$ ). Again, excluding the few indifferent participants, the difference between the two scenarios is highly significant $(p<.001)$.

Finally, for the third pair of scenarios, in which the winning team won $2-0$, the participants were equally divided about who was the better forecaster when the two goals

Table 2

Number of Participants Preferring Lower (Mr. Landman) or Higher (Mr. Peters) Forecasts as a Function of Early or Late Scores by the Winning Team

\begin{tabular}{cccc}
\hline & \multicolumn{3}{c}{ Preferred Forecaster } \\
\cline { 2 - 4 } Result & $\begin{array}{c}\text { Mr Landman } \\
\text { (Low Estimates) }\end{array}$ & $\begin{array}{c}\text { Mr Peters } \\
\text { (High Estimates) }\end{array}$ & $\begin{array}{c}\text { No } \\
\text { Difference }\end{array}$ \\
\hline 1-0 & $\frac{60 \%}{14}$ & $\frac{75 \%}{27}$ & 5 \\
A1: Goal early & 31 & 12 & 4 \\
A2: Goal late & $\underline{70 \%}$ & $\frac{80 \%}{25}$ & \\
3-2 & 11 & 12 & 2 \\
B1: Goals early & 36 & $90 \%$ & 3 \\
B2: Goals late & $75 \%$ & 21 & 3 \\
2-0 & 20 & 14 & 1 \\
C1: Goals early & 33 & & \\
C2: Goals late & & 73 & 10 \\
Total & 45 & 38 & 8 \\
Goals early & 100 & & \\
Goals late &
\end{tabular}

Note-Percentages refer to the probability of the outcome according to each forecaster. were scored at the 25th and 60th minutes but had a clear preference for the forecaster with the lower probability judgment (Mr. Landman, $p=.75$ ) when the goals were scored toward the end of the game. The difference between the two scenarios is statistically significant $(p<.05)$.

There is a clear and consistent pattern underlying the results in the three scenarios. Evidently, as is predicted by the correspondence hypothesis, the sooner the uncertainty (regarding the winner) is resolved, the higher the justified probability estimate (in retrospect). When the team is predicted to win scores early in the game, it is as if its winning potential has materialized, and this impression remains through the entire game. In contrast, uncertainty throughout the entire game is encoded as if the winning potential is not as strong (indeed, scoring toward the end may be perceived as incidental ${ }^{6}$ ) and consequently is associated (in hindsight) with a lower probability.

The previous two experiments indicate that probabilityoutcome correspondences are related to two different outcome measures: margin of winning and temporal pattern of scores. The following experiments were conducted to further test the robustness and generality of these results in a different domain - namely, with the prediction of earthquakes. In these experiments, we asked the participants to compare probabilistic predictions of earthquakes in a given region, issued by two earthquake experts. In all the scenarios, we assumed that an earthquake actually took place, but with a stronger or weaker intensity (Experiment 3 ) or within a shorter or longer time period after the prediction (Experiments 4 and 5). According to our hypothesis, the participants would prefer the higher probability both with stronger and with earlier eruptions, as compared with weaker and later eruptions, showing probability-outcome correspondence with respect to both intensity and time.

\section{EXPERIMENT 3}

\section{Method}

Participants. The participants were 62 students from the University of Utrecht, who volunteered to participate in this and other decision-making tasks, for which they were paid 11 Dutch guilders (approximately \$6).

Design and Procedure. The participants were introduced to a scenario regarding predictions of an earthquake in a given area, made by two expert forecasters (the full text is presented in the Appendix). One forecaster, George, predicted an earthquake within the coming 3 years with a probability of $60 \%$. His colleague, Steve, predicted the same event with probability of $80 \%$. The participants were further told that an earthquake indeed occurred 12 months from the day the two forecasters made their predictions. Half of the participants were told that the magnitude of the earthquake, as measured on the Richter scale, was 1.5 (low-magnitude condition); the other half were told that the magnitude of the earthquake was 6.5 (high-magnitude condition). Given that information, the participants were asked to judge which of the two forecasters made a better prediction. According to the probability-outcome correspondence hypothesis, the majority of the participants in the low-magnitude condition should judge George (who made the low-probability forecast) to be the better forecaster. Similarly, the majority of participants in the high-magnitude condition should judge Steve (who made the higher probability forecast) to be the better forecaster. 
Table 3

Number of Participants (With Percentages) Preferring the Lower $(60 \%)$ or Higher $(80 \%)$ Probability Forecast as a Function of Earthquake Intensity

\begin{tabular}{cccccc}
\hline & \multicolumn{3}{c}{ Preferred Forecaster } \\
\cline { 2 - 3 } $\begin{array}{c}\text { Intensity of } \\
\text { Earthquake }\end{array}$ & \multicolumn{2}{c}{ George (60\%) } & & \multicolumn{2}{c}{ Steve (80\%) } \\
\cline { 2 - 3 } & $n$ & $\%$ & & $\%$ \\
\hline $\begin{array}{c}\text { Condition 1 } \\
\text { Low (1.5) } \\
\text { Condition 2 } \\
\text { High (6.5) }\end{array}$ & 11 & 35.5 & 20 & 64.5 \\
\hline
\end{tabular}

\section{Results}

The results are shown in Table 3. As can be seen, in both conditions, there was a larger proportion of participants who preferred the higher probability forecaster (Steve). The main effect of forecaster (i.e., high vs. low probability) was significant $(p<.001)$. More essential, there was also a significant interaction ( $p<.001$, as indicated by a onetailed Fisher's exact test) between earthquake intensity and preferred forecaster, as is predicted by the correspondence hypothesis.

\section{EXPERIMENT 4}

\section{Method}

Participants. The participants were 80 volunteer students recruited at different points on the campus of the Free University of Amsterdam. They were approached by an experimenter, who asked whether they would be willing to participate in a short experiment, which would last less than 5 min.

Design and Procedure. The participants were presented with a scenario similar to the one employed in Experiment 3. The two forecasters, George and Steve, predicted the occurrence of an earthquake within the next 3 years to be $60 \%$ and $80 \%$, respectively. The independent variable in this experiment was the time of occurrence: Half the participants (early condition) were told that the earthquake occurred 3 months after the predictions were made; the other half (late condition) were told that the earthquake occurred 2 years and 7 months after the predictions were made. The magnitude of the earthquake for both groups was set equal at 3 on the Richter scale. Again, the participants' task was to judge who was a better forecaster. According to the correspondence hypothesis, more participants should judge George to have been the better forecaster under the late condition than under the early condition.

\section{Results}

As can be seen from Table 4A, the majority of the participants $(69 \%)$ in this experiment have a preference for George, who made the more cautious forecast of $60 \%$. More important, a larger proportion of participants prefer George under the late condition, as compared with the early condition. As is predicted by the correspondence hypothesis, there was a significant interaction $(p<.05$, using Fisher's exact test) between probability forecast and time of earthquake occurrence. Evidently, as in the previous experiment, predicted outcomes that materialize earlier are judged (other things being equal) to warrant higher probabilities.

\section{EXPERIMENT 5}

Experiment 4 assessed preferred probability (dependent variable) as a function of time of occurrence (independent variable). In contrast, Experiment 5 was designed to test the correspondence between probability and time in the other direction by varying the forecaster's probability estimate and asking the participants to predict the time of occurrence. Here, time of occurrence served as the dependent variable, and level of probability served as the independent variable. Hence, the purpose of Experiment 5 was to further substantiate the correspondence between probability and the temporal dimension.

\section{Method}

Participants. The participants were 78 volunteer students recruited, as in Experiment 4, at different points on the campus of the Free University of Amsterdam.

Design and Procedure. The participants were presented with a scenario similar to that employed in Experiment 4. They were provided with probability estimates of a single expert: For half of the subjects, the expert predicted an earthquake within the coming 3 years with a probability of $60 \%$ (low condition); for the other half, this probability was set at $80 \%$ (high condition). Both groups were then told that an earthquake indeed occurred, with a strength of 3 on the Richter scale, as in Experiment 4. The participants were asked to judge whether it was more likely that the earthquake occurred 3 months after the prediction (early eruption) or 2 years and 7 months after the prediction (late eruption).

\section{Results and Discussion}

The results are portrayed in Table 4B. Congruent with the results of Experiment 4, the correspondence hypothesis is again confirmed: In the high-probability (80\%) condition, approximately an equal number of participants

Table 4A

Number of Participants (With Percentages) in Experiment 4 Preferring the Lower $(60 \%)$ or Higher $(80 \%)$

Probability Forecast as a Function of Time Elapsed Before Earthquake Eruption

\begin{tabular}{cccccc}
\hline & \multicolumn{3}{c}{ Preferred Forecaster } \\
\cline { 2 - 3 } \cline { 5 - 6 } $\begin{array}{c}\text { Eruption of } \\
\text { Earthquake }\end{array}$ & $n$ & George (60\%) & & \multicolumn{2}{c}{ Steve (80\%) } \\
\cline { 2 - 3 } \cline { 5 - 6 } & $n$ & & & \\
\hline $\begin{array}{c}\text { Condition 1 } \\
\text { Early }\end{array}$ & 23 & 57.5 & & 17 & 42.5 \\
$\begin{array}{c}\text { Condition 2 } \\
\text { Late }\end{array}$ & 32 & 80.0 & & 8 & 20.0 \\
\hline
\end{tabular}

\section{Table 4B}

Number of Participants (With Percentages) in Experiment 5 Predicting an Early or Late Eruption of Earthquake, as a Function of Low $(60 \%)$ or High $(80 \%)$ Probability

\begin{tabular}{|c|c|c|c|c|}
\hline \multirow{3}{*}{$\begin{array}{l}\text { Probability } \\
\text { of Earthquake }\end{array}$} & \multicolumn{4}{|c|}{ Predicted Eruption of Earthquake } \\
\hline & \multicolumn{2}{|c|}{ Early } & \multicolumn{2}{|c|}{ Late } \\
\hline & $n$ & $\%$ & $\bar{n}$ & $\%$ \\
\hline $\begin{array}{l}\text { Condition } 1 \\
\text { Low }(60 \%)\end{array}$ & 8 & 20.5 & 31 & 79.5 \\
\hline $\begin{array}{c}\text { Condition } 2 \\
\text { High }(80 \%)\end{array}$ & 20 & 51.3 & 19 & 48.7 \\
\hline
\end{tabular}


predicted an early and a late eruption. In contrast, in the low-probability $(60 \%)$ condition, a large majority predicted a late eruption. The difference between the two conditions was highly significant $(p<.005$, by a Fisher exact test). Evidently, the correspondence between probability and temporal occurrence constitutes a two-way relationship.

The results of the last two experiments in combination with Experiment 2, in addition to corroborating the correspondence hypothesis, are compatible and complementary to previous approaches that examined the relationship between time and uncertainty. For instance, Prelec and Loewenstein (1991) delineate the resemblance between decision making over time and under uncertainty, and Keren and Roelofsma (1995) provide empirical evidence for the correspondence between time delay and uncertainty. The later show a systematic relationshipin which delayed prospects are associated with higher uncertainty and higher risk. Rachlin, Castrogiovanni, and Cross (1991; see also Rachlin \& Siegel, 1994) go as far as proposing that time delay and uncertainty are virtually the same and, further, that probability is a derivative of time delay. Although the exact nature of the relationship between time delay and uncertainty remains controversial (Keren \& Roelofsma, 1995), there is little doubt that these two facets are deeply interrelated, as is postulated by the correspondence hypothesis.

Experiments 1-5 provide accumulating evidence in support of the probability-outcome correspondence conjecture. According to this hypothesis, a probability statement can serve as an indicator of the (potential) underlying power(s) that may be responsible for the event under consideration. There is an isomorphic relationship between the probability and the strength by which the event will be manifested. A probability statement thus conveys, at the same time, two types of information: the likelihood that the event will occur and the strength by which it will occur.

Suppose that one is certain about the occurrence of the event and, accordingly, assigns it a probability of 1.0. How would one be able to convey possible differences in the magnitude of the relevant event? One possibility is to use probabilities higher than one. Indeed, casual observations suggest that, in colloquial speech, probabilities that are higher than one are not uncommon. What does one mean by saying, for instance, that "I am 200\% sure that she will pass the exam." Such a statement leaves no doubt about the occurrence of the event; yet, one may wonder why the speaker has not used $100 \%$, which, as far as likelihoods are concerned, conveys the same information?

The answer, we propose, lies in the logic of conversation (Schwartz, 1996). Communicators usually provide relevant information that gives neither too much nor too little information and attempt to produce a message that is appropriate to their communicative intent and to the specific context (McCann \& Higgins, 1992). Accordingly, we suggest that probabilities higher than 1.0 have an additional informational value-namely, expressing the strength of the relevant underlying disposition (or propensity). Hence, the statement in the example above implies not just certainty that she will pass the exam, but rather that she has the potential of getting a very high grade, much beyond just passing the exam. The following experiment was designed to test this conjecture.

\section{EXPERIMENT 6}

\section{Method}

Participants. The participants were 54 students from the University of Utrecht, who volunteered to participate in this and other decision-making tasks, for which they were paid 12 Dutch guilders (approximately \$6).

Design and Procedure. The participants were told about two basketball fans, Bruno and Karl, both of whom predicted that Italy would win an upcoming game against France. Bruno said that he was $100 \%$ certain that Italy would win; Karl said that he was $200 \%$ certain. Half of the participants were told that, in fact, Italy had won with a score of 88-42; the other half were told that Italy had won with a score of 78-67. After hearing the results, Bruno and Karl both claimed that they were perfectly right and that they could not have made a better prediction. The participants were asked to judge who of the two sounded more convincing.

\section{Results and Discussion}

The results are portrayed in Table 5 . As is predicted by the correspondence hypothesis, the majority of our participants believed that $200 \%$ was more convincing when the score was 88-42 (a real defeat), whereas most believed that $100 \%$ was more convincing when the score was 78-67. A chi-square test shows that the difference is highly significant $\left[\chi^{2}(1, N=54)=7.50, p<.01\right]$. Evidently, probabilities higher than $100 \%$ are associated with extreme outcomes.

These results were further corroborated in a small-scale experiment with different participants, who were presented with the following statement: "Today it rained non-stop the whole day. Early in the morning I told Peter that I am 200\% $(60 \%)$ certain that it's going to rain. Evidently, I was right." For half of the participants, $p=200 \%$, and for the other half, $p=60 \%$, and both groups were asked to rate (on a scale of 1-7 where $1=$ completely reasonable and meaningful) the statement in terms of "how reasonable and meaningful it was." Participants in the $200 \%$ condition $(\bar{x}=3.41)$ rated the statement as more reasonable than did the participants in the $60 \%$ condition $(\bar{x}=4.92)$, a difference that was statistically significant $(p<.01)$.

We do not suggest that the participants failed to understand the fundamental prescription that probabilities should never exceed $100 \%$. Rather, we propose that prob-

Table 5

Number Of Participants in Experiment 6 Preferring the Higher $\mathbf{2 0 0 \%}$ ) or the Lower (100\%) Probability Assessment as a Function of the End Score

\begin{tabular}{ccc}
\hline End Score & $200 \%$ & $100 \%$ \\
\hline $88-42$ & 17 & 10 \\
$78-67$ & 7 & 20 \\
\hline
\end{tabular}


abilities larger than $100 \%$ are used metaphorically to express not just the certainty of the outcome, but in addition its expected magnitude. In this respect, the results of Experiment 6 should be viewed as a generalization of those of Experiments 1-5.

\section{GENERAL DISCUSSION}

In the present article, we have shown that the way lay people interpret and understand probabilistic statements goes beyond what is intended by the formal theory. Apparently, probabilities are not just likelihood indicators, as is assumed by the formal theory. Evidently, they are perceived as containing additional information. Specifically, they serve as indicators of the extent to which an event will be fully realized: Larger probabilities do not just imply a higher likelihood of occurrence, but also signify the strength and power by which the event will be manifested. This is the essence of the probability-outcome correspondence principle.

Probability-outcome correspondences can often (correctly) be observed in daily life. A weather forecast involving a slight chance of rain may frequently be followed by small amounts of precipitation, whereas high probabilities of rain may be more predictive of heavy showers. Our empirical findings show that such correlations are indeed noticed and used by people as a criterion for evaluating probabilities. Sometimes, however, people generalize and apply the correspondence principle (unjustifiably) to situations in which the correlation is weak or nonexistent. In addition, we have shown that the correspondence principle is applied in both directions, and thus people seem willing to endorse a probability of $200 \%$ just to make it correspond to a rather extreme outcome.

An overall account of our findings is best achieved by a lay theory in which probabilities are conceived (and perceived) as reflecting the disposition and potency underlying the event under discussion. Constructs such as potential, propensity, or disposition are unobservable and are very similar to underlying traits (which are also unobservable) that can supposedly explain human behavior, as is postulated by implicit theories of personality (Chiu et al., 1997). In our proposed framework, as well as in implicit personality theories, inferential processes are based on viewing the predicted event (behavior, in implicit personality theories) as reflecting its corresponding disposition. Knowledge derived from the disposition serves as the basis for predicting the relevant event (behavior, in implicit personality theories). Unlike implicit personality theories, in the present framework, we assume dispositions that do not necessarily remain stable.

The impetus to interpret probabilities as propensities or dispositions was motivated by Popper's $(1959,1990)$ proposal, according to which inherent in each physical situation is a certain tendency of an event to be realized. Others have also employed the idea of dispositions or propensities as reflecting the internal representation of probabilities. For example, Kahneman and Tversky (1982) refer to external uncertainty (which is attributed to the real world) as reflecting dispositions. The term disposition, in this context, is interpreted as a characteristic of the relevant system. They further classify external uncertainty according to whether it is assessed in a distributional mode based on frequencies or by a singular mode, "in which probabilities are assessed by the propensities of the particular case in hand" (p. 152).

Our own explication of the term propensity is very similar to that proposed by Kahneman and Tversky (1982). Consider, for instance, the prediction of the outcome of a soccer game. To make such a prediction, one can rely on two types of information. One, which is distributional (frequentistic) in nature, would be to consult data of previous games between the two relevant teams. The benefits that may be obtained from such data, however, are limited. Soccer teams continuously change players, coaches, and management, and hence, their competence is dynamically changing. The other alternative, and the one we believe is employed by most soccer fans, is to employ the singular (story-based) mode and assess the propensity of each team to win. Such an approach (which is subjective and causal in nature) would require what Kahneman and Tversky (1982) term an inside view analysis. In our example, it would entail subjective assessments of the quality of the individual players of the two teams, the fitness of the key players, the number of injured players, the quality of the coach, who the home team is and what kind of supporters it has, and so forth. Combining all these factors (and possible interactions between them) results in an assessment of the potential strength or potency inherent in each team.

It is this strength or propensity underlying the case at hand that determines the corresponding probability. ${ }^{7} \mathrm{We}$ propose that the internal presentation of a probability judgment, adopted by lay people, is formed in terms of the corresponding propensity or disposition. Although a direct test of such an assertion is obviously impossible, we formulated a somewhat weaker hypothesis-namely, the probability-outcome correspondence. The cognitive representation of a probability statement that we allude to is founded on the (perceived) propensity of a causal system to result in particular outcomes (Kahneman \& Varey, 1990).

According to the implicit or intuitive model we envisage, probability statements can be viewed as predicting outcomes, on the one hand, and as reflecting underlying tendencies or propensities, on the other hand. Since probabilities vary in magnitude, from 0 to 1.0 , it is natural to think that these numbers somehow reflect the strength of the underlying tendencies. With categorical outcomes, they will simply indicate the tendency for a particular outcome to occur, as has been shown by Keren and Teigen (2001). With quantitative outcomes, they should also be related to characteristics of the predicted outcomesnamely, providing information about the strength or magnitude of the predicted event. In this respect, a probability statement can be considered to mirror the propensity, which in turn is believed to have a larger or smaller causal potency to yield a stronger or weaker effect. We thus as- 
sume that people think in terms similar to a causal power theory, which Cheng (1997) described as "the intuitive notion that one thing causes another by virtue of the power or energy that it exerts over the other" (p. 368). Indeed, White (1989) has suggested that this is the manner by which people typically interpret cause-effect relationships. We propose that this kind of reasoning occurs also in cases in which the causal agent is implicit, rather than observable.

What are the implications of the framework proposed in the present article, and what insights does it provide for a better understanding of probabilistic reasoning of lay people? In the following sections, we briefly comment on possible links with previous research on probabilityjudgments.

Weber (1994) has noted that earlier theories of choice behavior assume that the utility of uncertain options could be described by combining the separate assessments of probability and outcome. She reviews empirical evidence that shows, at the descriptive level, the existence of dependencies between probability and outcome evaluation. The empirical results reported in the present article provide additional evidence for such dependencies.

The propensity interpretation of probability statements is compatible with formal probability calculus; yet, the intuitive notion of probability-outcome correspondence may lead to probability judgments that are not always warranted by normative theory. First, our studies show that people use this principle to draw inferences about outcome characteristics that go beyond the standard interpretation of probabilities. For instance, a high probability of an earthquake is believed to predict the occurrence of an early or a particularly strong eruption. Second, these same outcome characteristics may be used as a heuristic device to (retrospectively) estimate the "most appropriate" probability (an early eruption is taken to mean that the earthquake must have had a high occurrence probability). Third, if probabilities mirror propensities, evidence for high or low propensities may be used heuristically for probability assessments, without due consideration of other formal constraints. In this respect, our model has much in common with the well-known representativeness heuristic (originally proposed by Kahneman \& Tversky, 1972). This heuristic is also based on a notion of correspondencenamely, that the sample's characteristics should correspond to the population from which it was drawn. More specifically, it postulates that the most probable sample is the one that most directly corresponds to the parent distribution and also to the process by which it is drawn.

Note, however, that the inferences drawn from our correspondence principle are essentially different from those implied by the representativeness heuristic. The representativeness heuristic is employed in order to infer probabilities, whereas the correspondence principle, as formulated in our framework, employs probabilities in order to infer outcome magnitude (and the time at which the outcome will occur).

A more direct illustration of how propensities can be used heuristically is to be found in a recent study by Sanbonmatsu, Posavac, and Stasney (1997). In one of their experiments, participants were asked to assess the probabilities for each of four qualified candidates to be offered a university position. In another experiment, all the candidates were described in more negative terms. In both cases, the participants seemed to disregard the requirement that probabilities should add up to 1.0 but gave generally very high estimates in the first condition and low estimates in the second. Although the authors interpreted their finding in a framework of selective, confirmatory hypothesis testing, it is clearly also compatible with the idea that indicators of disposition strength (implied by the descriptions of the randomly drawn candidates) can be used as a probability heuristic, overriding formal rules of probability.

A potency interpretation of probabilities can also be meaningfully applied to a puzzling finding reported by Windschitl and Weber (1999). In their studies, participants were presented with numeric expert forecasts-for instance, that a student with previous low grades (Cs) has a $70 \%$ chance of passing her next exam. Another group was given the same probability forecast about a student with higher grades (Bs). Despite the identical probabilities, the chances of the second student were rated to be better (she would quite likely pass the exam) than the former (she would be somewhat likely to pass the exam). Windschitl and Weber described this as a context effect, based on the "representativeness" of the predicted events. We suggest that the predictions were based on the candidates' perceived propensities (abilities) of passing an exam. These propensities could be inferred from the numerical probabilities, but also from previous grades. It follows that the probabilities assigned to a talented student are somehow more promising than the same probability assigned to a less powerful candidate.

Another finding that is easily interpreted in dispositional (propensity) terms and serves as another demonstration of the correspondence principle is reported by Weber and Hilton (1990). Employing medical scenarios, these authors showed that numerical interpretations of probability words were, among other things, dependent on the severity of the outcome predicted to occur. As is entailed by the correspondence principle, more severe outcomes were associated with higher probabilities.

The idea that people perceive the world in terms of dispositions (which do or do not unfold) is certainly tenable in a more general form. We have already mentioned implicit theories of personalities, in which traits represent the disposition of a particular individual. Similarly, many of the studies of naive physics suggest that people conceive physical events to be the result of potential unobservable energy. Indeed, the strong tendency that we all have to explain the world around us in some causal terms assumes an implicit or explicit generative source (containing certain propensities) with which observed outcomes can be explained (White, 1989). Medin (1989) has recently proposed a framework, which he termed psychologicalessentialism, according to which "people act as if things (e.g., objects) have essences or underlying natures that make 
them the things they are" (p. 1476). What all these approaches have in common is the idea of inherent, often unobservable, mechanisms that are responsible for the observed outcome(s).

Note that all these approaches also assume a certain kind of causal reasoning, an assumption that is not shared by the traditional interpretations of probability. For instance, the frequentistic approach is based on a match between the different possible outcomes and their corresponding frequencies and is thus correlational in nature. It implies a mapping of frequencies into a single number (called probability), in which causality has no place. The descriptive framework presented here, based on the idea of dispositions and potency, entails an underlying process of causal reasoning. This suggests that it could prove fruitful to explore the links between probabilistic thinking and causal reasoning schemas.

\section{REFERENCES}

Cheng, P. W. (1997). From covariation to causation: A causal power theory. Psychological Review, 104, 367-405.

Chiu, C., Hong, Y., \& Dweck, C. (1997). Lay dispositionismand implicit theories of personality. Journal of Personality \& Social Psychology, 73, 19-30.

Coombs, C. H., Dawes, R. M., \& Tversky, A. (1970) Mathematicalpsychology: An elementary introduction. Englewood Cliffs, NJ: PrenticeHall.

Howell, W. C., \& Burnett, S. A. (1978). Uncertainty measurement: A cognitive taxonomy. Organizational Behavior \& Human Performance, 22, 45-68.

Kahneman, D., \& Tversky, A. (1972). Subjective probability: A judgment of representativeness. Cognitive Psychology, 3, 430-454.

Kahneman, D., \& Tversky, A. (1982). Variants of uncertainty. Cognition, 11, 143-157.

Kahneman, D., \& VArey, C. A. (1990). Propensities and counterfactuals: The loser that almost won. Journal of Personality \& Social Psychology, 59, 1101-1110.

Keren, G., \& Roelofsma, P. (1995). Immediacy and certainty in intertemporal choice. Organizational Behavior \& Human Decision Processes, 63, 287-297.

Keren, G., \& Teigen, K. H. (2001). Why is $p=.90$ better than $p=.70$ ? Preference for definitive predictions by lay consumers of probability judgments. Psychonomic Bulletin \& Review, 8, 191-202.

McCAnN, C. D., \& Higgins, E. T. (1992). Personal and contextual factors in communication: A review of the "communication game." In G. R. Semin \& K. Fiedler (Eds.), Language, interaction, and social cognition (pp. 144-172). Newbury Park, CA: Sage

McCloskey, M. (1983, November). Intuitive physics. Scientific American, 24, 122-130.

Medin, D. L. (1989). Concepts and conceptual structure. American Psychologist, 44, 1469-1481.

MiLL, J. S. (1856). A system of logic. London: Parker.

POPPER, K. (1959). The propensity interpretation of probability. British Journal for the Philosophy of Science, 10, 25-42.

Popper, K. (1990). A world of propensities: Two new views of causality. Bristol: Thoemmes.

Prelec, D., \& Loewenstein, G. (1991). Decision making over time and under uncertainty: A common approach. Management Science, 37, 770-786.

Rachlin, H., Castrogiovanni, A., \& Cross, D. (1991). Probability and delay in commitment. Journal of the Experimental Analysis of Behavior, 48, 347-353.
Rachlin, H. \& Siegel, E. (1994). Temporal patterning in probabilistic choice. Organizational Behavior \& Human Decision Processes, 59 , 161-176.

Salmon, W. C. (1967). The foundationof scientific inference. Pittsburgh University of Pittsburgh Press.

Sanbonmatsu, D. M., Posavac, S. S., \& Stasney, R. (1997). The subjective beliefs underlying probability overestimation. Journal of Experimental Social Psychology, 33, 276-295.

Schwartz, N. (1996) Cognition and communication. Mahwah, NJ: Erlbaum.

Shafer, G. (1993). Can the various meanings of probability be reconciled? In G. Keren \& C. Lewis (Eds.), A handbookfor data analysis in the behavioral sciences: Methodological issues (pp. 165-198). Hillsdale, NJ: Erlbaum.

Teigen, K. H. (1994). Variants of subjective probabilities: Concepts, norms, and biases. In G. Wright \& P. Ayton (Eds.), Subjective probability (pp. 211-238). Chichester: Wiley.

WEBER, E. U. (1994). From subjective probabilities to decision weights: The effect of asymmetric loss functions on the evaluation of uncertain outcomes and events. Psychological Bulletin, 115, 228-242.

Weber, E. U., \& Hilton, D. (1990). Contextual effects in the interpretations of probability words: Perceived base-rate and severity of events. Journal of Experimental Psychology: Human Perception \& Performance, 16, 781-789.

White, P. A. (1989). A theory of causal processing. British Journal of Psychology, 80, 431-454.

WinDSCHITL,P. D., \& WeBER, E. U. (1999). The interpretation of "likely" depends on the context, but " $70 \%$ " is 70\% —right?: The influence of associative processes on perceived certainty. Journal of Experimental Psychology: Learning, Memory, \& Cognition, 25, 1514-1533.

Wright, G., \& Ayton, P. (EDS.) (1994). Subjective probability. Chichester, U.K.: Wiley.

YAtes, J. F., Price, P. C., Lee, J. W., \& Ramirez, J. (1996). Good probabilistic forecasters: The "consumer's" perspective. International Journal of Forecasting, 12, 41-56.

\section{NOTES}

1. Coombs, Dawes, and Tversky (1970) correctly noted that "despite their different natures, descriptive and normative theories are deeply interrelated in most applications."

2. In the present paper, the terms propensity and disposition are used interchangeably.

3. It is important to note that Popper $(1959,1990)$ rejected the subjective interpretation of probabilities that he believed to be untenable. However, he also expressed strong reservations with regard to the traditional frequentistic interpretation and introduced the concept of propensities in order to account for probabilities of single events.

4. Note that, whenever available, we do not exclude the use of frequentistic data for assessing the propensity of the relevant event.

5 . As a further test, we examined a subset of the games resulting in a $1-0$ score, in which the difference in the ranking between the two teams at the time of the game (obtained from their standing in the league) was at least six ranks. When the moment at which the goal was scored was used as the dependent variable, there was no difference between those games in which the higher ranking team won and those in which the lower ranking team won.

6. One possibility is that when a goal is scored only in the last minute, it is much easier to construct counterfactuals in which the team did not win than when the goal was scored early in the game and the score was maintained through the entire game.

7. The concept of propensity encapsulated in an object, event, or situation is, in our view, not limited to the singular mode (in this respect, we depart from Kahneman \& Tversky, 1972). A frequentistic analysis may similarly lead to an assessment of disposition, a view that is compatible with Popper's analysis. 
APPENDIX

Instructions to Participants in the Different Experiments

\section{Experiment 1}

We are interested in the evaluation of forecasters. Below are the forecasts of two basketballexperts, named Peter and John, regarding four different games. Before the games took place, each expert was required to state the name of the winning team according to his opinion, and in addition to estimate the probability that the team he named will indeed win the game (for example, "70\% Blues" means that the forecaster believes that Blues will win with a confidence of $70 \%$ ). The actual results are given in the last column. As you may note, both forecasters believed that the home teams would win and had 3 (out of 4 ) correct forecasts.

\begin{tabular}{llccccc}
\hline & & & & \multicolumn{3}{c}{ Outcome } \\
\cline { 5 - 6 } Home team & Guest team & Peter & John & Condition 1 & Condition 2 & Condition 3 \\
\hline The Blues & Eagles & $70 \%$ Blu. & $90 \%$ blu. & Blu. won & $93: 71$ & $84: 80$ \\
Goldenball & Bears & $70 \%$ Gol. & $90 \%$ Gol. & Gol. won & $74: 54$ & $65: 63$ \\
Angels & Sportclub & $70 \%$ Ang. & $90 \%$ Ang. & Ang. lost & $86: 88$ & $59: 76$ \\
Universal & Lions & $70 \%$ Uni. & $90 \%$ Univ. & Univ. won & $81: 58$ & $76: 73$ \\
\hline
\end{tabular}

Please look carefully on the predictions of each forecaster and the corresponding outcomes. We would like you to decide which of the two forecasters made better predictions [made more informative predications]. Note: There is no "correct" answer and we simply want to have your opinion.

* Peter made better probability forecasts.

* John made better probability forecasts.

* Peter and John made equally good forecasts.

\section{Experiment 2}

Consider the following situation: Mr. Peters and Mr. Landman are both soccer experts. During their careers they have been employed by several newspapers covering soccer games for the sport section and in addition were hired as experts by different soccer teams. Both claim that they are superb forecasters and can predict with high accuracy the outcomes of different games. They are currently involved in a prestigious competition of predicting the outcomes of games. The competition is conducted as follows: A week before a game takes place, each of them is required to predict which of the two teams will win. In addition, they have to make a probability judgment (on a scale between $50 \%$ and 100\%) reflecting how certain they are that the team they chose will indeed win the game. After the game, their probabilistic predictions and the corresponding outcomes are given to an independent judge. For each game, the judge has to determine who of the two forecasters made a better prediction.

Your task in this experiment is to serve as the judge who determines for each prediction who was the better forecaster, Mr. Peters or Mr. Landman. On each of the following three pages you will receive the predictions made by the two experts and information about the outcome. Your task in each case is to determine who was the better forecaster.

Note: To avoid any prejudices by the judge, we label the names of the teams by capital letters.

\section{Condition A1 (early)}

For the game between Team $\mathbf{A}$ and Team $\mathbf{B}$ the following forecasts were made:

Mr Landman predicted that team A will win with probability of $60 \%$.

Mr Peters predicted that team A will win with probability of $75 \%$.

Outcome: It was an enjoyable game to watch with some very exciting moments. The score at half time was 1:0 for team $\mathrm{A}$ as a result of a single goal scored on the 8th minute. There were no further goals and thus team A won $1: 0$.

Who made a better probability judgment: Mr. Peters or Mr. Landman?

* Mr. Peters made a better judgment.

* Mr. Landman made a better judgment.

Condition A2 (late) (identical to A1 except for outcome):

Outcome: It was an enjoyable game to watch with some very exciting moments. The score at half time was a tie of 0:0. At the end, team A won 1:0 as a result of a single goal scored on the 89th minute, just before the game was over.

\section{Condition B1 (early)}

For the game between Team $\mathbf{C}$ and Team $\mathbf{D}$ the following forecasts were made:

Mr Landman predicted that team D will win with probability of $70 \%$.

Mr Peters predicted that team $\mathbf{D}$ will win with probability of $80 \%$.

Outcome: Both teams played an ambitious game that was accompanied by many tackles. At half time team D was leading 3:0 after scoring three times at the 3rd, 12th, and the 39th minute. The second goal was scored after a wonderful combination between three players. After the break the game became tougher and team $\mathrm{C}$ scored twice, just before the game was over, on the 86th and 90th minutes. The end score was thus 3:2 in favor of D. 


\section{APPENDIX (Continued)}

Condition B2 (late) (identical to B1 except for outcome):

Outcome: Both teams played an ambitious game that was accompanied by many tackles. At half time team $\mathrm{C}$ was leading 2:0 after scoring two times at the 12th, and the 39th minute. The second goal was scored after a wonderful combination between three players. After the break the game became tougher and team D scored three times, just before the game was over, on the 79th, 86th and 90th minutes. The end score was thus 3:2 in favor of D.

\section{Condition C1 (early)}

For the game between Team $\mathbf{E}$ and Team $\mathbf{F}$ the following forecasts were made:

Mr Landman predicted that team $\mathbf{E}$ will win with probability of $75 \%$.

Mr Peters predicted that team $\mathbf{E}$ will win with probability of $90 \%$.

Outcome: It was a game for which supporters of both teams were waiting for a long time and was indeed an exciting game. The score at half time was 1:0 for team $\mathrm{E}$ as result of a single goal scored on the 25 th minute. A second goal for team $\mathrm{E}$ was scored at the 60th minute as a result of which Team E won 2:0

Condition C2 (late) (identical to $\mathrm{C} 1$ except for outcome):

Outcome: It was a game for which supporters of both teams were waiting for a long time and was indeed an exciting game. The score at half time was a tie of 0:0. Close to the end of the game team E scored twice, in the 81 st and 85 th minute as a result of which Team E won 2:0.

\section{Experiment 3}

Consider two expert geologists, George and Steve, who after long investigations of a specific district predict the likelihood of an earthquake in the district under investigation. It is known that during the 19th century there were a few earthquakes in the region, ranging from very weak to mildly strong ones. On the basis of their recent investigations (in which they used the most sophisticated available technology) they make the following predictions:

-George predicts that "The probability of an earthquake in the district within the coming 3 years is $60 \%$ ". - Steve predicts that "The probability of an earthquake in the district within the coming 3 years is $80 \%$ ". The strength of an earthquake is measured on a Richter scale which varies between 0 and approximately 9 , where the larger the number the stronger the earthquake. As a matter of fact, an earthquake occurred 12 years from the day they made their predictions, and it measured 1.5 (6.5) on the Richter scale. Who, according to your judgment, made a better probabilistic prediction, Peter or John? (there is no "correct" answer and we are just interested in your personal opinion).

* Steve made a better probability judgment.

* George made a better probability judgment. 